\title{
Multifunctional PID Neuro-Controller for Synchronous Generator
}

\author{
Hocine Tiliouine \\ Gdańsk University of Technology, Faculty of Electrical and Control Engineering, Gdańsk, Poland \\ e-mail: hocine.tiliouine@pg.gda.pl
}

Keywords: PID Neuro-Controller, conventional PID controller, self-tuning control, synchronous generator.

\begin{abstract}
This paper deals with a PID Neuro-Controller (PIDNC) for synchronous generator system. The controller is based on artificial neural network and adaptive control strategy. It ensures two functions: maintaining the generator voltage at its desired value and damping electromechanical oscillations. The performance of the proposed controller is evaluated on the basis of simulation tests. A comparative study of the results obtained with PIDNC and those with conventional PID was performed.
\end{abstract}

\section{Introduction}

With the constantly increasing demand for electric power industry, the structure of electrical power network becomes more and more complex, making it difficult to exploit and exposed more frequently to the risk of disturbances. Therefore, the problem of the control in this field continues to be of great importance and draws the attention of many searchers $[9,10,11]$.

The synchronous generators that are part of the electrical power network, represent highly nonlinear systems, which parameters and mode of operation change with time. Regulators with constant parameters, such as the conventional PID controller [4,5], used in the excitation control of these generators are unable in some cases of disturbances to ensure stable operation. Thus, to improve the performance control, new techniques based on artificial intelligence are proposed. Among them, neural networks are relatively suitable to overcome these difficulties. Because of its self-learning and nonlinear mapping ability, they are successfully utilized in the field of control and modeling [6-8].

In this paper an adaptive PID control system for a synchronous generator connected to an infinite-bus power system is presented. The proposed control system performs two functions simultaneously: maintaining the voltage of the alternator at its desired value and damping of electromechanical oscillations.

\section{Proposed control system}

The structure of the proposed control system is shown in figure 1. The control scheme consists of two neural networks: the first named $\mathrm{CN}$ and whose outputs are the PID controller parameters and the second named NNE used to identify the plant to be controlled. The signal $Y_{r e f}$ is a vector whose components are the reference voltage $U_{r e f}$ and the reference active power $P_{r e f}$. However the output $Y$ is a vector whose components are the terminal voltage $U_{g}$ and the active power $P_{g}$ of the synchronous generator.

The control method is based on the adaptive control strategy $[3,4]$ that can be described by the following algorithm:

$$
u(k)=u(k-1)+k_{P}(e(k)-e(k-1))+k_{I} e(k)+k_{D}(e(k)-2 e(k-1)+e(k-2))
$$

where $u(k), e(k)$ represent respectively the control signal and the sampling error at time $k$. The error $e(k)$ is expressed as:

$$
e(k)=\left(U_{r e f}-U_{g}(k)\right)+\left(P_{r e f}-P_{g}(k)\right) .
$$

The coefficients $k_{P}, k_{I}, k_{D}$ are the PID controller parameters. 


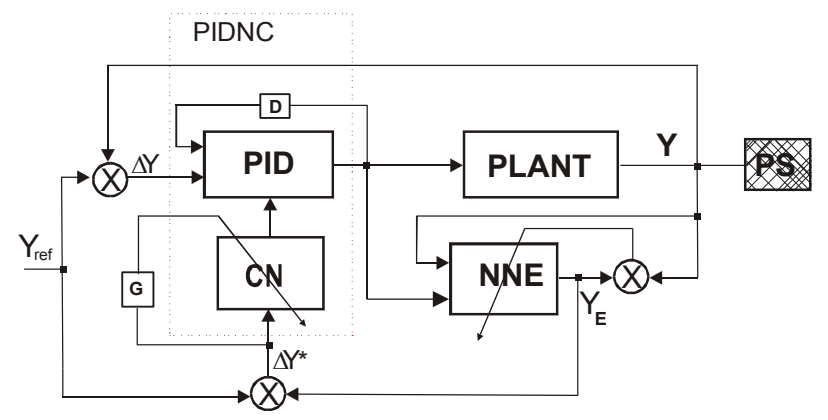

Figure 1. Block diagram of the proposed control system.

\section{A. Pid neuro-controller structure}

The PID Neuro-Controller named PIDNC has a simple structure as described in fig.2. It includes just a single two layers neural network, named $\mathrm{CN}$, with $\mathrm{m}=9$ hidden units. The input vector $\Delta Y^{*}$ is equal to the vector deviation $\left[Y_{E}-Y\right]^{T}$ where $Y_{E}=\left[U_{E}, \mathrm{P}_{\mathrm{E}}\right]^{T}$ represents the output vector of the emulator and $Y=\left[U_{g}, \mathrm{P}_{\mathrm{g}}\right]^{T}$ the output vector of the plant as shown in fig.1. All neurons of the network are nonlinear, with a sigmoid activation function $f\left(s_{j}\right)=1 /\left(1+e^{-s_{j}}\right)$, where $s_{j}=\sum_{i} w_{i j} x_{i}$. To each input signal $x_{i}$ of neuron $j$ is assigned a weight $w_{i j}$. The three outputs of the neural network are the tuned parameters $k_{p}, k_{i}, k_{D}$ used in the PID algorithm given in equation (1).

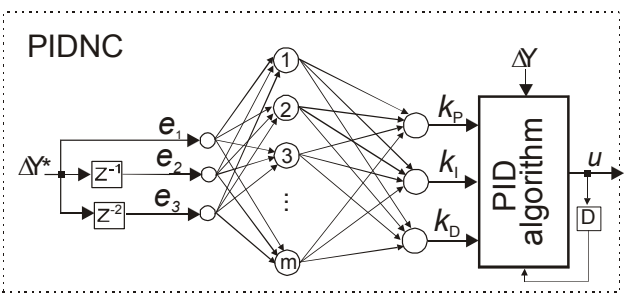

Figure 2. Structure of the PIDNC.

\section{B. Neural network emulator structure}

The neural network named NNE is used to identify the plant. Its inputs are the delayed outputs and the actual and delayed inputs of the plant. The outputs $U_{E}, P_{E}$ of the NNE are the predicted values of the plant outputs.

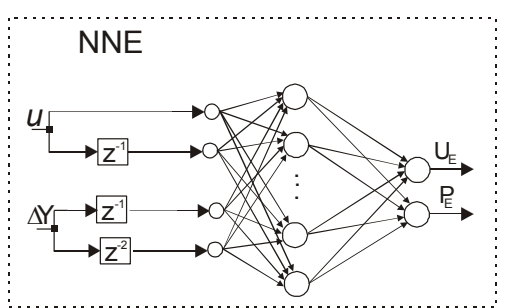

Figure 3. Structure of the NNE.

\section{On-line learning algorithm}

To adjust the weights of the neural networks $\mathrm{CN}$ and NNE a gradient descent method is adopted [1,2].

Let $w_{i j}$ denote the weight associated with the link originating from neuron $i$ towards neuron $j$ at time $k$. The adjustment of that weight is expressed as:

$$
w_{i j}(k+1)=w_{i j}(k)+\eta\left(-\frac{\partial J(k)}{\partial w_{i j}(k)}\right)
$$


where $\eta$ represents the learning rate and $J$ the performance function. The function $J$ is defined by:

$$
J(k)=\frac{1}{2} \sum\left(y_{r e f}(k)-y(k)\right)^{2}
$$

where $y_{\text {ref }}(k), \mathrm{y}(k)$ represent respectively the desired values and the real plant value output values. For the proposed control system components oh these vectors are respectively $U_{\text {ref, }} P_{\text {ref }}$ and $U_{g}(k), P_{g}(k)$. The learning objective is to minimize the performance function $J$.

The gradient of the function $J$ with respect to an arbitrary weight vector $w_{i j}$ is expressed by:

$$
\begin{aligned}
\frac{\partial J(k)}{\partial w_{i j}(k)}= & \frac{\partial}{\partial w_{i j}}\left(\frac{1}{2} \sum\left(y_{r e f}(k)-y(k)\right)^{2}\right. \\
= & \left(\sum\left(y_{r e f}(k)-y(k)\right)\left(-\frac{\partial y(k)}{\partial w_{i j}}\right)\right. \\
& =\left(\sum\left(y_{r e f}(k)-y(k)\right)\left(-\frac{\partial y(k)}{\partial u(k)} \frac{\partial u(k)}{\partial w_{i j}}\right)\right.
\end{aligned}
$$

\section{Simulation tests}

In order to evaluate the performance of the proposed PID Neuro-Controller, simulation tests have been carried out for a single synchronous machine connected to an infinite bus power system.

Figure $4 \mathrm{a}$ and figure $4 \mathrm{~b}$ show the system responses to step changes in reference voltage. At time $t=0 \mathrm{~s}$ and at $t=5 \mathrm{~s}$ the reference voltage is changed respectively to $105 \%$ and $95 \%$ value of the rated voltage. Figure $5 \mathrm{a}$ and figure $5 \mathrm{~b}$ illustrate the system responses to a three-phase shortcircuit in infinite bus occurred at time $t=0 \mathrm{~s}$ and cleared at $t=150 \mathrm{~ms}$.

The simulation results show that the generator voltage is recovered faster with the proposed controller (PIDNC) than with conventional PID controller (CPID). However, the oscillation damping effect is roughly the same for the two types of controllers.

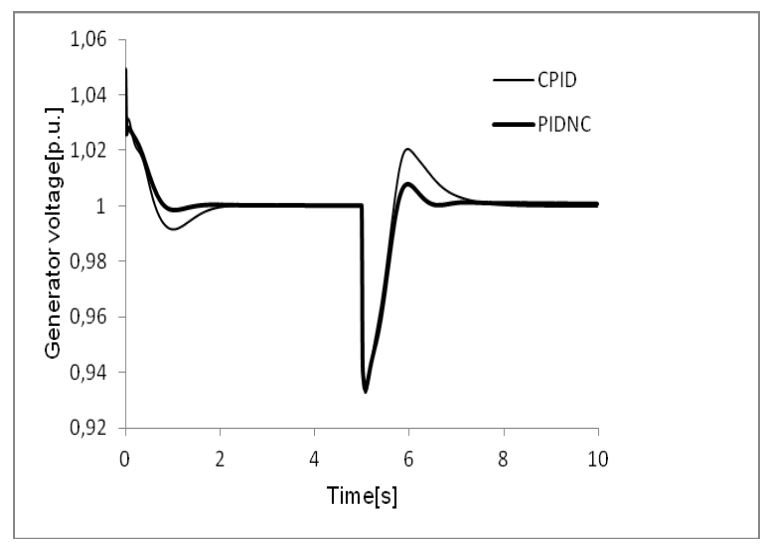

(a)

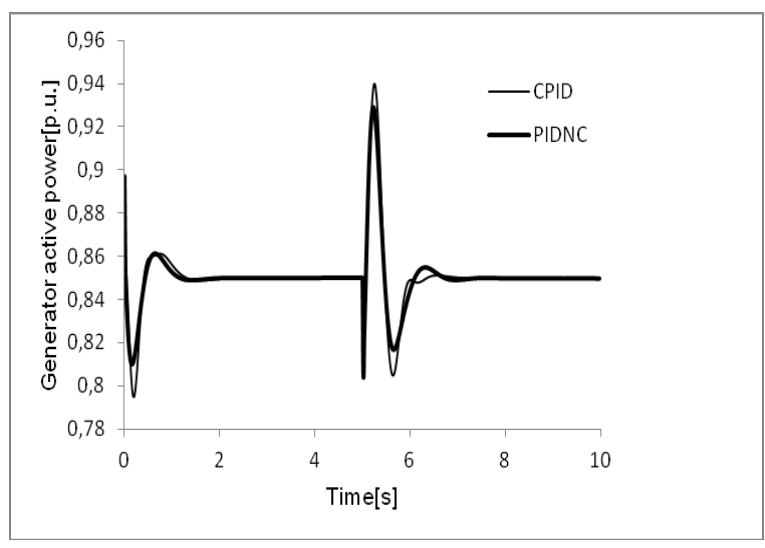

(b)

Figure 4. System response to a $\pm 5 \%$ step change in reference voltage. 


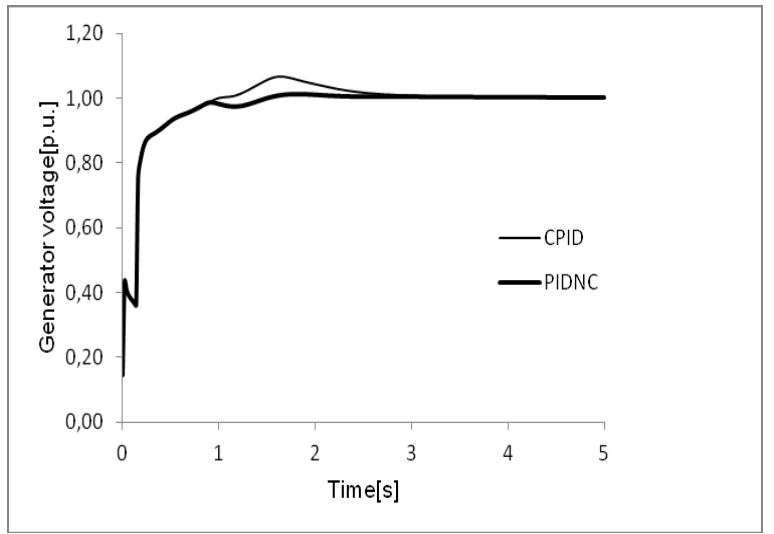

(a)

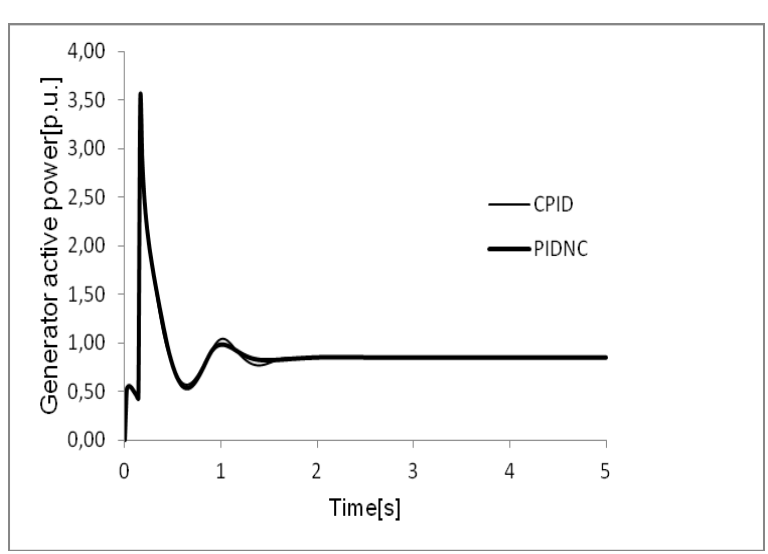

(b)

Figure 5. System response to a three-phase short-circuit.

\section{Conclusions}

A multifunctional PID Neuro-Controller (PIDNC) for synchronous generator has been presented. It performs two functions: as a voltage controller and as a power system stabilizer. Its PID parameters are tuned according to the generator operating conditions by a feedforward artificial neural network named NC.

The effectiveness of the proposed PIDNC was evaluated through diverse simulation tests. The obtained results show that in comparison with a conventional PID controller, the PIDNC is more efficient.

\section{References}

[1] M.M. Gupta, L. Jin, N. Homma, Static and dynamic neural networks, from fundamentals to advanced theory, IEEE PRESS, A John Wiley \& Sons, Inc., Hoboken, New Jersey, 2003.

[2] H. Tiliouine, Comparative study of neural networks used in modeling and control of dynamic systems, Przegląd Elektrotechniczny (Electrical Review). 87(7) (2011) 104-109.

[3] K.J. Wstrom, T. Hagglund, PID controllers: Theory, design, and tuning, Triangle Park, NC, ISA, 1995.

[4] T. Gang, Adaptive control design and analysis, John Wilej \& Sons, New Jersey, 2003.

[5] K. Ang, G. Chong, Y. Li, PID control system analysis, design and technology, IEEE Transaction on Control Systems Technology. 13(4) (2005) 559-576.

[6] G.W. Irwin, K. Warwick, K.J. Hunt, Neural network applications in control, Institution of Electrical Engineers, London, 1995.

[7] O. Sigeru, K. Marzuki, Y. Rubiyah, Neuro-control and its applications, Advances in industrial control, Springer-Verlag, London, 1995.

[8] P. Shamsollahi, O.P. Malik, An adaptive power system stabilizer on-line trained neural networks, IEEE Transactions on Energy Conversion. 12(4) (1997) 382-387.

[9] L. Wen, L. Gao, D. Yiping, Generating unit and control system model for stability analysis of power system, Consumer Electronics, Communications and Networks (CECNet), 2011 International Conference on, IEEE, 2011, pp.382-386.

[10] H. Zhao et al., Excitation prediction control of multi-machine power systems using balanced reduced model, IET Generation, Transmission \& Distribution. 8(6) (2014) 1075-1081.

[11] A. Saleem, M. Lind, S.N. Singh, Modeling control situations in power system operations, Autonomous and Intelligent Systems (AIS), International Conference on, pp.1-6, 2010. 